\title{
Fluorescence imaging inside an internal combustion engine using tunable excimer lasers
}

\author{
Peter Andresen, Gerard Meijer, Harald Schlüter, Heiner Voges, Andrea Koch, Werner Hentschel, \\ Winfried Oppermann, and Erhard Rothe
}

\begin{abstract}
Tunable excimer lasers are used to obtain 2-D images of molecular (and some state-specific) density distributions inside a cylinder of a modified four-cylinder in-line engine that has optical access. Natural fluorescence (i.e., without a laser) is used for some $\mathrm{OH}$ pictures, normal laser-induced fluorescence (LIF) for those of NO and of the isooctane fuel, and laser-induced predissociative fluorescence (LIPF) for other $\mathrm{OH}$ pictures and for those of $\mathrm{O}_{2}$. Relevant spectroscopy is done to find the laser and fluorescence frequencies needed to measure isolated species. LIPF works well at high pressures, is state specific, and is ideally suited to follow turbulent processes. No similar measurements in engines have been previously reported. Pictures are taken in succeeding engine cycles. Their sequence is either at a particular point of the engine's cycle to show cyclic fluctuations, or at succeeding portions of the cycle to illustrate the progress of the gasdynamics or of the combustion.
\end{abstract}

\section{Introduction}

Lasers offer many desirable features for application to combustion diagnostics. ${ }^{1}$ They allow fine control over the spatial and temporal extent of the species to be measured.

Laser techniques are particularly promising when combined detector and computer technologies are applied to combustion. These should, for example, lead to optimization of fuels and minimization of pollutants by appropriate control and feedback technology.

We have previously ${ }^{2}$ discussed some of the fundamentals and application of a new approach, i.e., laserinduced predissociative fluorescence (LIPF), parts of which were originally described by Massey and Lemon, ${ }^{3}$ for investigating turbulent high temperature reacting gases, such as occur in flames and in automobile cylinders or in low temperature media such as wind tunnels. When fully developed, this approach should yield precise and instantaneous (i.e., within $\approx 20 \mathrm{~ns}$ )

\footnotetext{
W. Hentschel and W. Oppermann are with Volkswagen AG Research Division, D-3180 Wolfsburg, Federal Republic of Germany; H. Voges and A. Koch are with Laser-Laboratorium-Göttingen e.V., Robert-Bosch-Breite 10, D-3400 Göttingen, Federal Republic of Germany; Erhard Rothe is with Wayne State University, Department of Chemical Engineering, Detroit, Michigan 48202; and the other authors are with Max-Planck-Institut fur Strömungsforschung, Bunsenstrasse 10, D-3400 Göttingen, Federal Republic of Germany.

Received 7 August 1989.

0003-6935/90/162392-13\$02.00/0.

(C) 1990 Optical Society of America.
}

distributions of constituent densities $n_{i}$ and of temperature $T$. Different applications of these ideas, mostly involving $\mathrm{O}_{2}$, are now also being pursued in several other laboratories. . $^{4-6}$

Here we describe measurements within a combustion chamber of a modified automobile engine using (a) LIPF, (b) normal laser-induced fluorescence (LIF), and (c) normal (i.e., not laser-induced) fluorescence (NF). They produced 2-D images of density distributions of $\mathrm{OH}, \mathrm{O}_{2}, \mathrm{NO}$, and iso- $\mathrm{C}_{8} \mathrm{H}_{18}$ within the cylinder. These images allow visualization of the gasdynamics and of the combustion process.

LIF imaging of $\mathrm{OH}$ in single-cylinder engines has been described by Felton et al. ${ }^{7}$ and by Schipperijn et $a l .8$

We had access to our engine for three weeks and all measurements described below were done in this time.

\section{Description of LIF and LIPF}

We use both LIF and LIPF. The use of normal LIF is not new. It is the most widely used laser technique to follow chemical processes that occur at pressures $<\approx 10^{-2}$ bar.

The advantage of LIPF is that it can be quantitatively and simply used at atmospheric and higher pressures such as those that exist, for example, with atmospheric flames or with internal combustion, where the interpretation of LIF depends on large, and probably unreliable, corrections.

In either LIF or LIPF, a laser beam passes through a medium and excites molecules from a state $i$ into a higher state $i^{*}$. Figure 1 has potential energy diagrams for the $X$ and $A$ states of $\mathrm{OH}$ and it will help to 


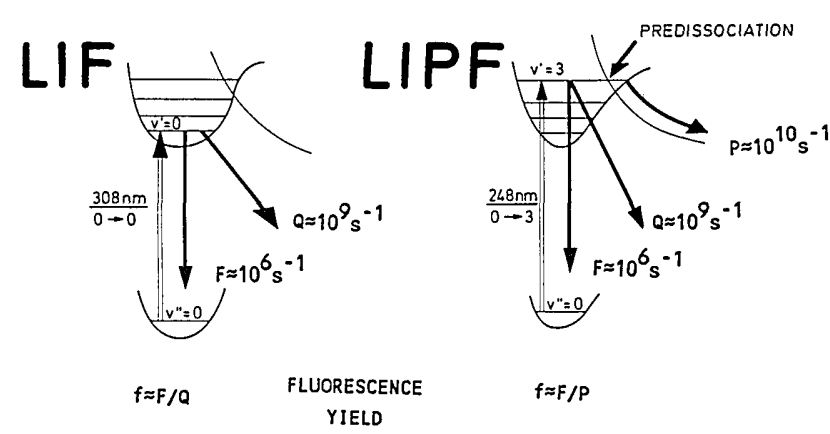

Fig. 1. Comparison of LIF and LIPF using the $A-X$ system of $\mathrm{OH}$ as an example. The value for $Q$ applies at 1 bar.

distinguish between LIF and LIPF. On the righthand side of Fig. 1 the excitation is from $v^{\prime \prime}=0(i)$ to $v^{\prime}$ $=3\left(i^{*}\right)$. The number of $i^{*}$ excited is proportional to $n_{i}$, the density in state $i$. State $i^{*}$ can (a) fluoresce, and be measured, with a rate $F$, or (b) predissociate, via the curve crossing, with rate $P$, or (c) quench via collisions with a rate $Q$.

The rates $F$ and $P$ depend only on the nature of the excited molecule, while the rate $Q$ also depends on gas composition, density and temperature, i.e., of the severity and the number of various types of collision. The perils of quenching were discussed in a review ${ }^{9}$ by Lucht. For example, at $1 \mathrm{~atm}$, such collisions quench almost all $i^{*}$ and, at any particular location, only a small fraction $f$ (typically $\approx 10^{-3}$ ) remains to emit light. Because $f$ depends strongly on the identity and the density of each constituent, and on the temperature, it is difficult to determine its absolute value. Even worse, because the composition and $T$ often change drastically within millimeters, $f$ will also change and as a result no self-consistent calibration is possible. This means that interpretation of LIF data depends on complex corrections. As described below, the beauty of LIPF is the essential absence of collisional quenching.

The intensity $I$ of the fluorescence is given by

$$
I=[F /(F+Q+P)] \theta n_{i}=f \theta n_{i},
$$

where $f=F /(F+Q+P)$ is the fraction of $i^{*}$ that fluoresces and $\theta$ is a constant that depends on molecular constants and on laser and other apparatus parameters. Fo.

If the goal is to measure $n_{i}$, we find $n_{i}=I(F+Q+P) /$

If $T$ is needed, we use two states with densities $n_{1}$ and $n_{2}$ and their known energies $E_{1}$ and $E_{2}$. These are related by $n_{1} / n_{2}=\left(g_{1} / g_{2}\right) \exp -\left[\left(E_{1}-E_{2}\right) / k T\right]$, where the $g_{i}$ are the statistical degeneracies, $2 j_{i}^{\prime \prime}+1$, and then

$$
T=\left[\left(E_{1}-E_{2}\right) / k\right] / \ln \left[\left(n_{2} / n_{1}\right)\left(g_{1} / g_{2}\right)\right] .
$$

Substituting from the expression for $n_{i}$ above, we obtain

$$
T=\left[\left(E_{1}-E_{2}\right) / k\right] / \ln \left\{[I(F+Q+P) / g F \theta]_{2} /[I(F+Q+P) / g F \theta]_{1}\right\} .
$$

From these equations we see that at low pressure, when $Q \ll F+P$, there is competition between fluorescence and predissociation, and at higher pressure there is also competition with quenching.

Two limiting cases are close to our conditions. These are:

Normal LIF at higher pressures. Normal LIF is shown at the left in Fig. 1 as a transition from $v^{\prime \prime}=0$ to $v^{\prime}=0$. There is no predissociation of $\mathrm{OH}$ from $v^{\prime}=0$ and so $P=0$. At high pressures, quenching predominates over fluorescence, so that $Q \gg F$. Then

$$
\begin{aligned}
& I=[F / Q] \theta n_{i}=f \theta n_{i}, \\
& n_{i}=I Q / F \theta, \\
& T=\left[\left(E_{1}-E_{2}\right) / k\right] / \ln \left[(I Q / g F \theta)_{2} /(I Q / g F \theta)_{1}\right] .
\end{aligned}
$$

This means that $I, n_{i}$, and $T$ all depend on the elusive $Q$. As noted above calibration is not possible.

Laser-induced predissociative fluorescence $(L I P F)$. Here $P \gg Q$ and $P \gg F$, so that

$$
\begin{aligned}
I & =[F / P] \theta n_{i}=f \theta n_{i}, \\
n_{i} & =I P / F \theta, \\
T & =\left[\left(E_{1}-E_{2}\right) / k\right] / \ln \left[(I P / g F \theta)_{2} /(I P / g F \theta)_{1}\right] .
\end{aligned}
$$

These equations show that $I, n_{i}$, and $T$ are not functions of $Q$, but only of the molecular constants $F$ and $P$. This means that calibration of the system can be done, and that interpretation is simple; i.e., $n_{i}$ is proportional to $I$.

Often a total molecular density $n\left(=\Sigma n_{i}\right)$ is desired rather than an individual $n_{i}$. From $n_{i}$ and $T$, we can calculate $n$ via the Boltzmann equation. For the greatest precision in $n$, it is best to select a state $i$ for which $n_{i}$ is fairly insensitive to $T$.

There are two basic ideas that make LIPF successful; these are (a) excitation to states that are so shortlived that there is no time for collisions and (b) the use of a powerful, tunable UV laser.

One might think that short-lived $i^{*}$ would lead to small $I$. However the lack of quenching, combined with the high spectral brightness of the laser, serves to yield signals that are comparable with those from LIF. For example, from the data in Fig. $1, f_{\mathrm{LIF}} \approx 10^{-3}$ and $f_{\text {LIPF }} \approx 10^{-4}$. Although $f_{\text {LIPF }}<f_{\mathrm{LIF}}$, the laser's high spectral brightness generates $I$ that are more than sufficient for imaging.

We use narrowband excimer lasers that provide UV light having four relevant features: (a) a high output energy in the $0.2-0.4-\mathrm{J} /$ pulse range, (b) narrow bandwidths, $\approx 0.2(\mathrm{KrF})$ and $\approx 0.5-\mathrm{cm}^{-1}(\mathrm{ArF}),(\mathrm{c})$ an $\approx 1-\mathrm{nm}$ tuning range, and $(d)$ a pulse width of $\approx 20 \mathrm{~ns}$. The spectral brightness is $100-1000$ times more than is available from a normal frequency doubled dye laser at these wavelengths.

Our present approach is well suited to combustion studies. Some of its advantages are that (a) large areas can be illuminated with efficient excitation; (b) even though the tuning range is only $\approx 1 \mathrm{~nm}$, three important combustion species, $\mathrm{OH}, \mathrm{O}_{2},{ }^{2-6,10}$ and $\mathrm{H}_{2} \mathrm{O},{ }^{11}$ can be analyzed via LIPF; (c) other molecules, such as $\mathrm{CO},{ }^{12} \mathrm{NO},{ }^{13}$ and hydrocarbons, are measurable with normal LIF, which means that important species from 


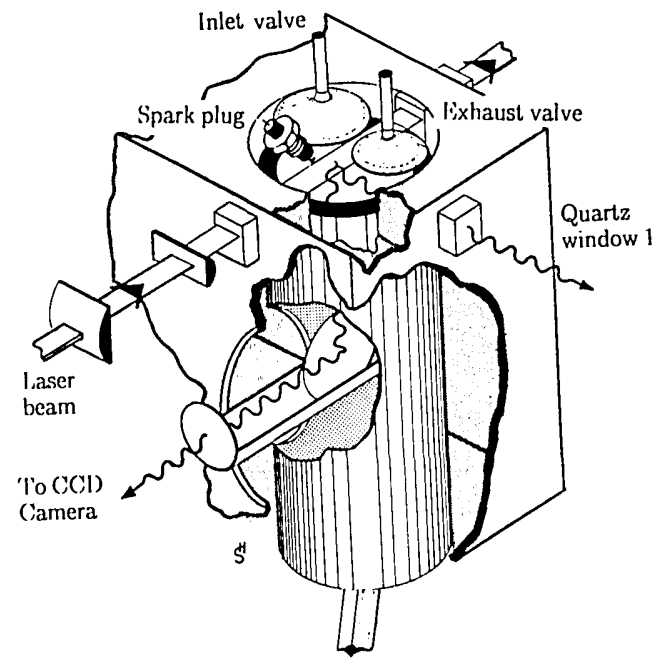

Fig. 2. View of the modified engine cylinder that shows the optical access.

the beginning $\left(\mathrm{O}_{2}\right.$, hydrocarbons), middle $(\mathrm{OH})$, and end ( $\mathrm{NO}, \mathrm{CO}, \mathrm{H}_{2} \mathrm{O}$ ) of the combustion process can be seen; (d) fluorescence from the detected molecules usually has a much different wavelength than that of the laser; this virtually eliminates scattered light problems; (e) the use of dispersed LIF, i.e., filtering of the fluorescence, allows detection of species in media where many species are simultaneously excited.

As discussed above, if two densities $n_{1}$ and $n_{2}$ are measured simultaneously at a point, $T$ can be calculated. To obtain a temperature field, the equation for $T$ is applied at each point. The 20-ns laser pulse provides superb time resolution and is thus ideal for analysis of turbulent burning.

\section{Experimental}

We use an in-line four-cylinder engine that is similar to a production model. It is mounted on a test-bed at the VW laboratories and it has modifications in its cylinders that allow optical access. We image its fourth cylinder. The arrangement is described below and is illustrated in Figs. 2-4. All windows and lenses used are made of UV transmitting Suprasil-I.

Figure 2 shows a schematic of the optical arrangement in the modified cylinder. At the top is the spark plug and the inlet and exhaust valves. The ribbonshaped laser beam passes about halfway between the cylinder head and the top of the piston's travel (i.e., the top dead center, TDC), so that the piston never blocks the beam. The laser light is shaped into the $0.4-\times 18$ $\mathrm{mm}$ ribbon by cylindrical lenses and then passes into, and out of, the cylinder through windows.

The lasers are oscillator-amplifier combinations (Lambda Physik EMG-160MSC-EST) that are operated on the $\operatorname{ArF}(\approx 193-\mathrm{nm})$ or on the $\mathrm{KrF}(\approx 248-\mathrm{nm})$ transitions. They are usually tuned to excite fluorescence from a particular quantum state of a desired species. Their pulse width is only $\approx 20 \mathrm{~ns}$, which is essentially instantaneous on the combustion time
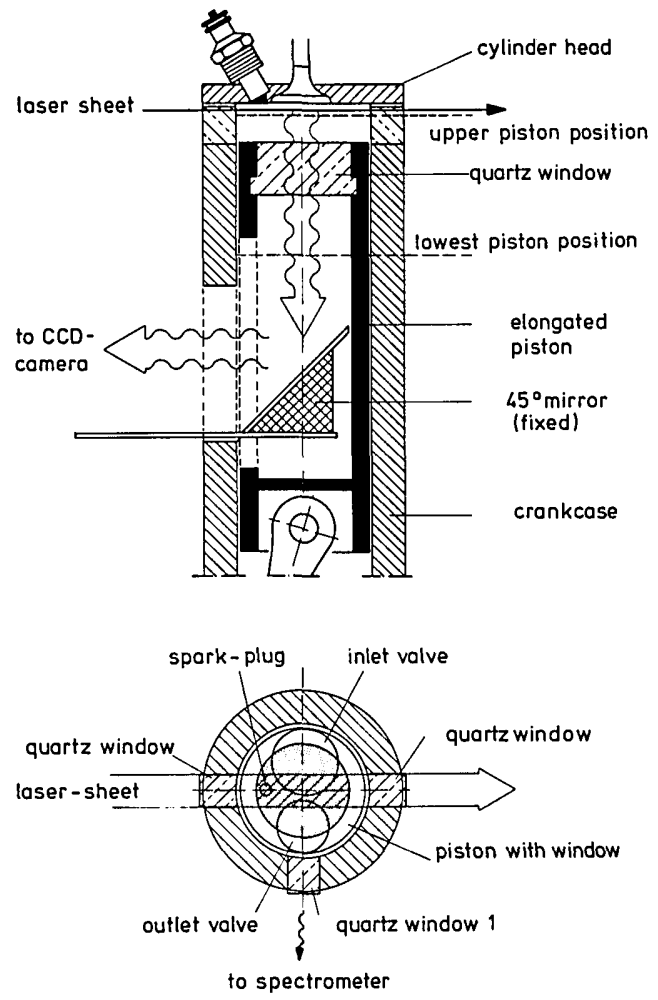

Fig. 3. Optical arrangement in the cylinder. The upper portion is a vertical cut, while the lower shows the laser path and the field of view of the camera.

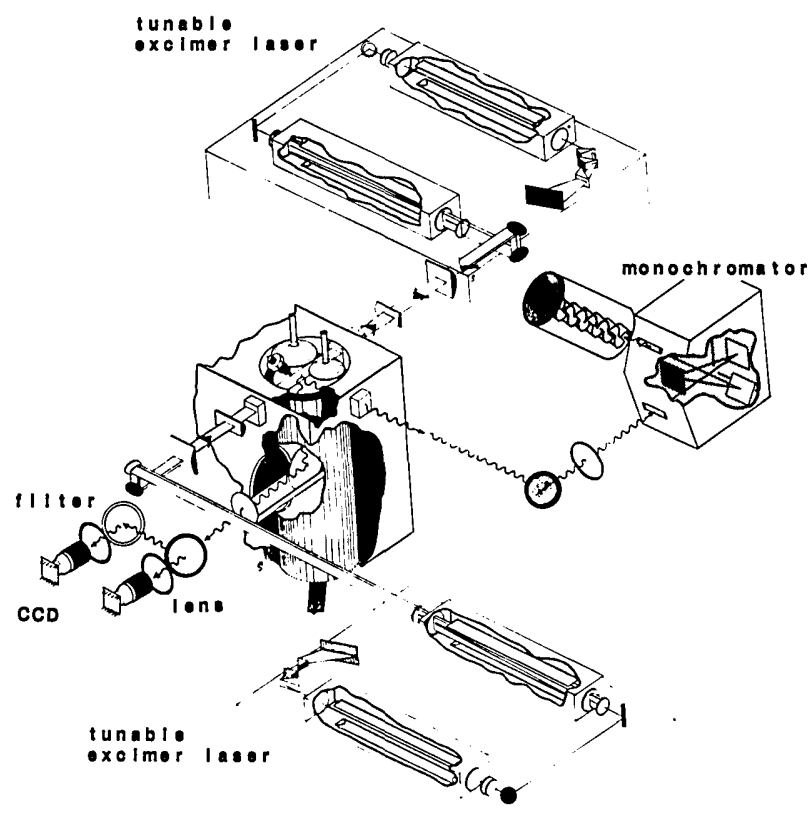

Fig. 4. Overall schematic of the experiment.

scale. Fluorescence leaves the cylinder through two different windows.

The light's exit through window 1 is direct. It is shown at the upper portion of Fig. 2 at $90^{\circ}$ to the laser beam. The light goes to a monochromator that dis- 
perses the fluorescence and so helps to properly tune the laser.

The fluorescence to be imaged passes through the other window, which is also the top of the piston. It is then reflected $90^{\circ}$ by a fixed mirror located beneath the piston. The piston is elongated so that its motion does not disturb the mirror. The light travels antiparallel to, and beneath, the laser beam to the imaging optics and camera. This arrangement is shown in Figs. 2 and 3.

The lower portion of Fig. 3 is a top view of the cylinder and helps to explain some of the images' features. The shaded circle is the camera's field of view. The fluorescence must also be within the laser sheet. That means that the bright area recorded by the camera is indicated by parallel diagonal lines that lie within the shaded area. The spark plug is at the left. The inlet valve and the somewhat smaller exhaust valve are at the top center and at the bottom center, respectively. These locations apply to all the camera's pictures, except Fig. 7, which is turned $90^{\circ}$ with the spark plug at the bottom.

Figure 4 shows some of the light being imaged onto cameras. Two lenses serve to make $\approx 3 \times$ size reduction. The first lens has a $300-\mathrm{mm}$ focal length and is used $300 \mathrm{~mm}$ from the laser ribbon. The resulting parallel light is sometimes filtered with dielectric mirrors that have a bandwidth of $\approx 15 \mathrm{~nm}$ and that have high reflectivity. The second lens, with a focal length of $100 \mathrm{~mm}$, serves to image the picture onto a camera system. A Schott UG11 camera filter, that transmits around $310-\mathrm{nm}$, is normally used with the $\mathrm{KrF}$ laser to reduce scattered $248-\mathrm{nm}$ light.

A camera system consists of an UV sensitive, high gain image intensifier that is coupled to a CCD camera by fiber optics. The intensifier is gated so that the camera normally records only $\approx 200 \mathrm{~ns}$ after each laser pulse. The resulting pictures are both (a) transcribed as an analog signal on a video cassette and (b) digitized in a computer and presented in false colors on an online monitor.

The fluorescence intensity $I$ is approximately (see previous section) proportional to the density of the desired species $n_{i}$ and false colors represent different intensities. The values for $I$ can be resolved into 256 (gray) levels. The relationship between the false colors and these $I$-levels is presented with the pictures. However, because of the expense of publishing color pictures, most of our images are black-and-white versions of the color shots. No effort is made to do the quenching corrections for the LIF or the NF data. However some results with $\mathrm{OH}$, and all with $\mathrm{O}_{2}$, were done with LIPF. If these had been corrected for the Beer's law absorption of the laser beam within the cylinder, they would have represented the quantitative spatial distribution of density. That absorption correction would not have been difficult but was not done. The images for fuel and NO were obtained from normal LIF and the remainder of the $\mathrm{OH}$ with $\mathrm{NF}$ and so these are inherently qualitative.

The fluorescence from window 1 is focused into a monochromator and the dispersed light is amplified with a photomultiplier. The output pulses go to a boxcar that averages ten laser pulses and sends the resulting signal to a strip chart recorder. The boxcar gate is typically $\approx 50 \mathrm{~ns}$. Fluorescence measurements with the monochromator and those with the camera involve different paths and measurement techniques, as well as spatial integration, and care must be applied in comparing their signals.

However the camera and monochromator are often used together. Then, as the laser is tuned, resonances appear on the recorder at the same time that the light at the camera brightens. This identifies the molecules, and their quantum states, that are being observed with the camera. It is this combination of (a) processing of weak light and (b) spectroscopic analysis that permits the 2-D determination of gas composition. The spectroscopic information also yields valuable information about the choice of camera filters.

Figure 4 is an overall apparatus schematic. Two tunable excimer lasers and two cameras are shown. At the right is the monochromator, at the left the cameras and their optics and filters. The fluorescence from each laser is separately recorded by a camera. The second laser and its camera are intended for temperature field measurements.

The firing of the laser, and the gates of the boxcar and of the image intensifier, are all triggered by the engine to occur at a fixed relationship to a preselected point of its cycle, i.e., of its crank angle (CA) which goes from $0-720^{\circ}$ as shown in Fig. 5 . We can take pictures at any desired CA.

The camera's pictures shown below are 2-D density distributions $n_{i}(x, y)$ that exist within the $\approx 20$-ns laser pulse, assuming (see, however, Sec. II) that $I$ is proportional to $n_{i}$. Because of this stop action the pictures capture the very turbulent evolution of the internal combustion. We measure either of two different types of picture sequence.

In one type, a fixed CA is selected and pictures are taken from many subsequent engine cycles. These can be analyzed for statistical fluctuations in $n_{i}(x, y)$. For example, we look at NO formation in succeeding cycles and derive information about the average locations, the fluctuations in that location, or about the fluctuation of $n_{i}$ at a particular location.

In the other sequence, the pictures are at increasing CA so that the progress of the gasdynamics or of the combustion process is seen. (These pictures are also from different cycles.) For example, we can see a flame front spread through the cylinder.

We use iso- $\mathrm{C}_{8} \mathrm{H}_{18}$ as the fuel because some components of a normal gasoline mixture absorb the excimer light so strongly that it does not go through the cylinder. The vapors of many saturated hydrocarbons do transmit near 193 and $248 \mathrm{~nm},{ }^{11}$ and so we expect that similar experiments can be done with other mixtures of saturated hydrocarbons. This would allow variation of the octane number in future tests.

Because tunable 193- and 248-nm excimer laser light had never been used within an engine cylinder, we 
Fig. 5. Basics of engine operation as a function of the crank angle. The upper half shows the piston and valve positions and the lower half has the cylinder pressure at $1000 \mathrm{rpm}$ and $20 \mathrm{Nm}$.
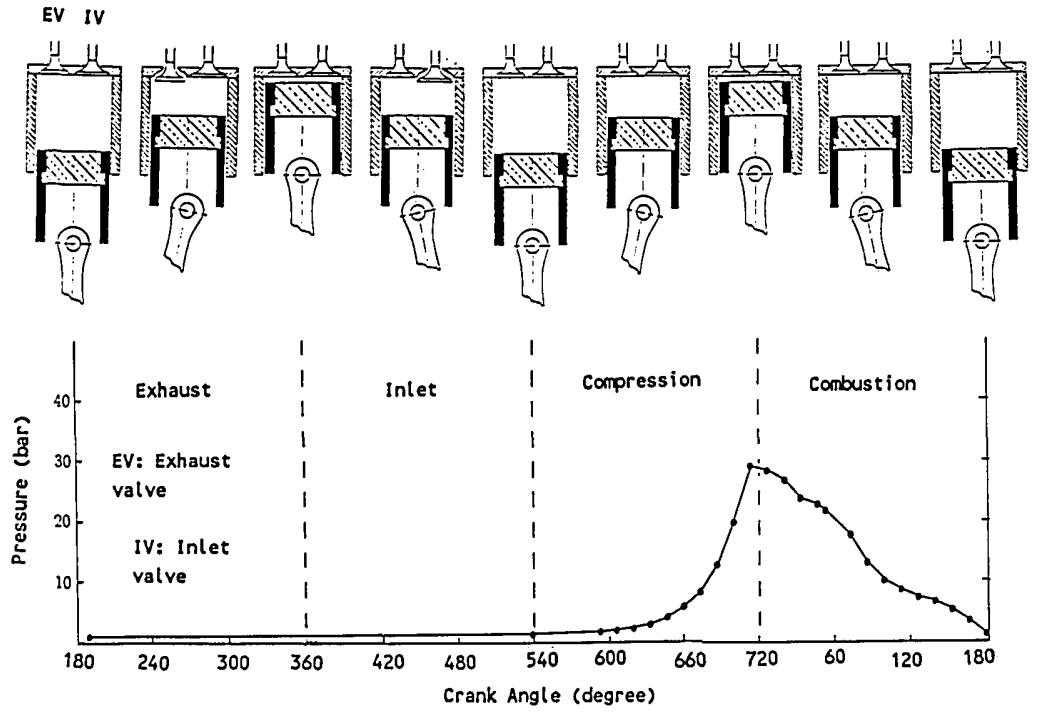

worried about potential problems. Some of these are that (a) the window might become opaque from deposition of fuel or of combustion products or (b) the high laser intensities could accelerate, or actually cause such deposition, or (c) the gas would be opaque at these short wavelengths, mixed compostions, high pressures, and high temperatures. Fortunately some of these fears are unfounded. For example, the engine runs for $\approx 30 \mathrm{~min}$ at a speed of $1000 \mathrm{rpm}$ before cleaning is necessary: this limit is caused by formation of thin lubricating oil films on the output mirror, and window, of the imaging optics. Some problems that do arise are circumvented by suitable changes of experimental conditions.

The engine's operating characteristics are described below with the help of Fig. 5. It shows the relationships of the piston positions, and the valves, as a function of CA as well as the corresponding cylinder pressures, which were measured at $1000 \mathrm{rpm}$. [The 1000 $\mathrm{rpm}$ means that the laser repetition rate is $1000 /(2 \times$ $60)=10.3 \mathrm{~Hz}$.]

Consider the nominal phases of the engine cycle. The piston is at its lowest point at the beginning $(\mathrm{CA}=$ $180^{\circ}$ ) of the exhaust phase. It then moves upward and pushes the combustion products through the exhaust valve. The inlet valve opens at $330^{\circ}$, just before the piston arrives at the TDC at $360^{\circ}$. The piston moves down during the inlet phase $\left(360-540^{\circ}\right)$ and fuel-air mixture comes in. Then the mixture is compressed $\left(540-720^{\circ}\right)$ and the spark plug ignites the mixture at $714^{\circ}$. Finally the power is delivered $\left(0-180^{\circ}\right)$ and the engine, at $180^{\circ}$, has completed its cycle. A maximum of $\approx 30$ bar is reached during the compression.

\section{Results}

\section{A. Density Distribution of Fuel from LIF}

When iso- $\mathrm{C}_{8} \mathrm{H}_{18}$ is excited by $248-\mathrm{nm}$ laser light, there is strong fluorescence. (There is little light from $193 \mathrm{~nm}$.) Figure 6 shows the dispersed fluorescence

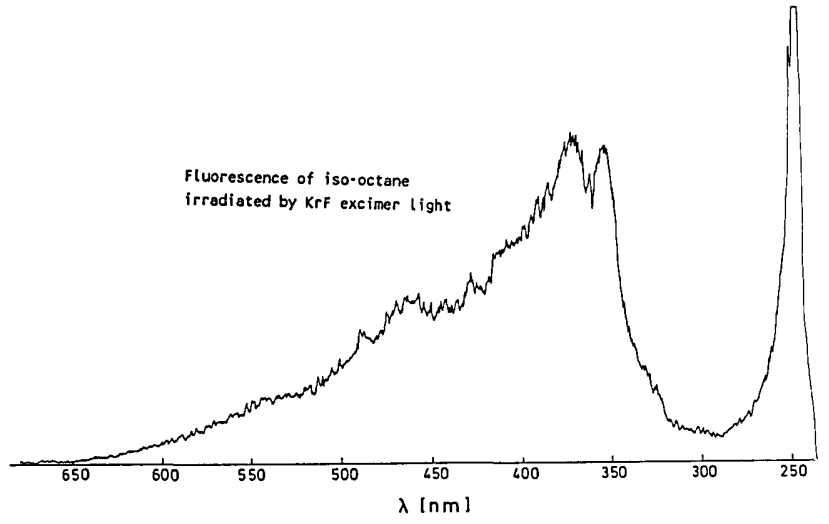

Fig. 6. Dispersion spectrum of a $300 \mathrm{~K}$ mixture of isooctane in air.

(dispersion spectrum) as measured in a separate experiment using a mixture of iso- $\mathrm{C}_{8} \mathrm{H}_{18}$ vapor and air at $\approx 1$ bar. This relatively unstructured spectum is essentially insensitive to laser tuning (within the $\mathrm{KrF}$ range) and it is used here to find $n_{\text {iso- } \mathrm{C}_{8} \mathrm{H}_{18}}(x, y)$.

Figure 7 shows a selection from a sequence of thirtyfour pictures that have $n_{\text {iso }-\mathrm{C}_{8} \mathrm{H}_{18}}(x, y)$ as a function of CA. Those on the bottom begin at the opening of the inlet valve and end after the time when the flame front has passed through the cylinder. Those at $\mathrm{CA}=330^{\circ}$ and $340^{\circ}$ are for the initial fuel-air intake. The fuel starts in a little before the inlet phase in Fig. 5. Remember that each picture is from a different cycle. The very different structures are caused by the turbulence in the fuel-air intake process. Later, in the inlet $\left(370^{\circ}\right)$ and compression $\left(650^{\circ}\right)$ phases, the distribution becomes more homogeneous. Those at $10^{\circ}, 30^{\circ}$, and $40^{\circ}$ are shortly after the spark plug fires at $-6^{\circ}$ $\left(714^{\circ}\right)$. In contrast to the other pictures, the plug is at the bottom in Fig. 7. As the combustion proceeds upward, it depletes the fuel and the region of high fuel concentration also moves up. The meager fluores- 

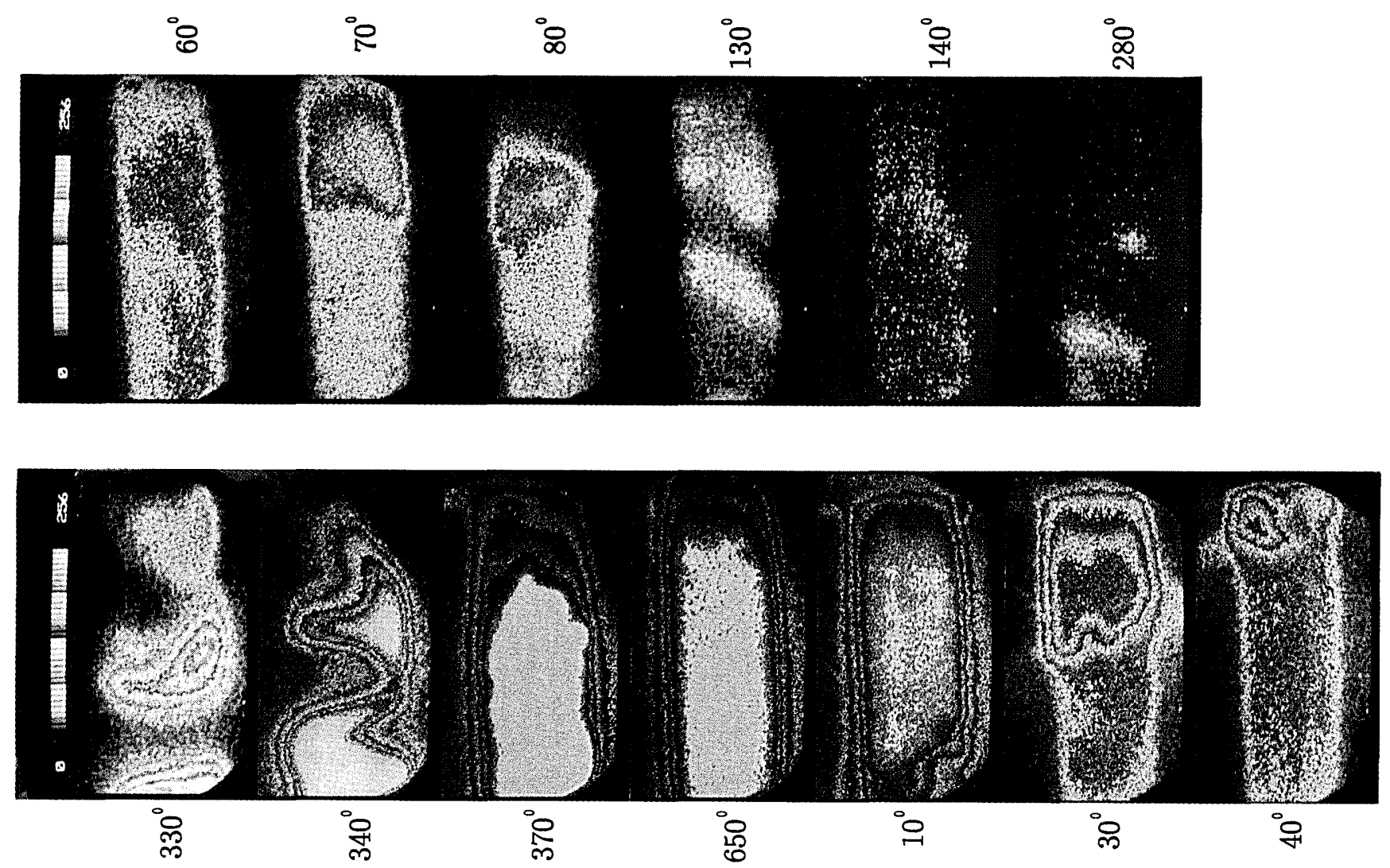

Fig. 7. Density distributions of iso-octane as a function of CA before, during, and after combustion. Ignition is at $714^{\circ}$. The spark plug is near the bottom.
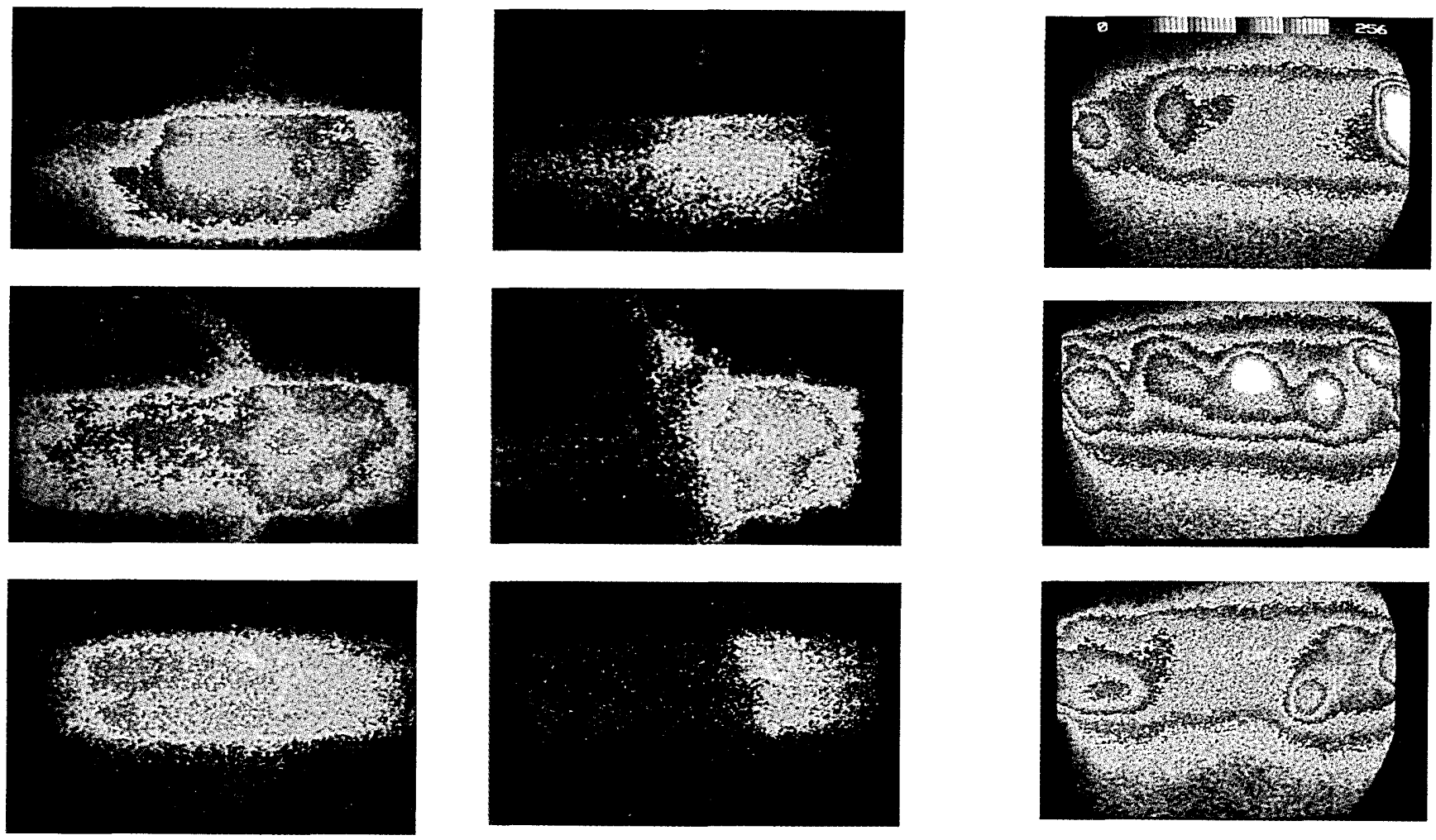

Fig. 15. Density distributions for state selected $\mathrm{OH}\left(\mathrm{v}^{\prime \prime}=0, \mathrm{~N}^{\prime \prime}=8\right)$ and NO $\left(\mathrm{v}^{\prime \prime}=1\right)$ at the left and right, respectively. See text for further description. 

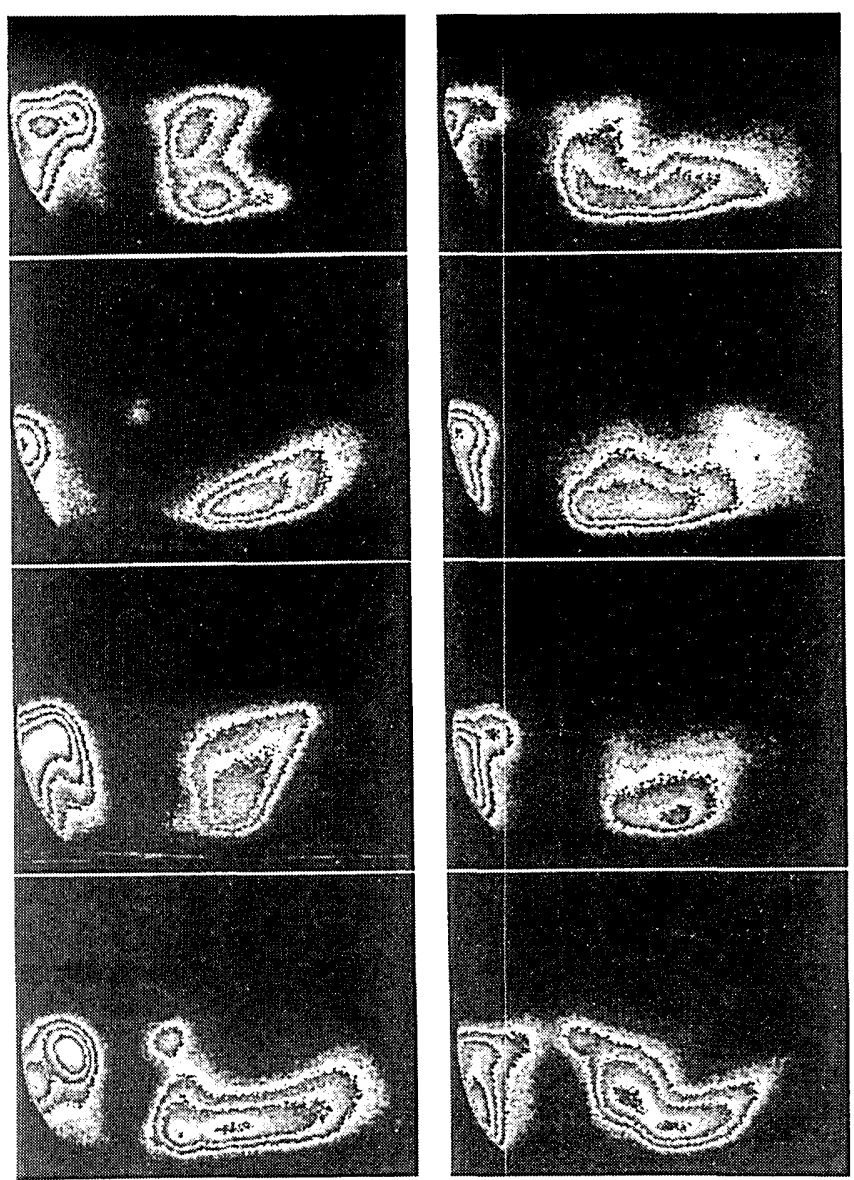

Fig. 8. Density distributions of isooctane in succeeding cycles at $\mathrm{CA}=330^{\circ}$ (i.e., in the early inlet phase). The intensity scale is the same as in Fig. 7.

cence at the bottom indicates the near absence of fuel there and it is this difference in intensity that makes the flame front visible.

In the $30^{\circ}$ and $40^{\circ}$ pictures there is still some fluorescence behind the flame front, which indicates some unburned fuel. This phenomenon is also seen at $60^{\circ}$, $70^{\circ}$, and $80^{\circ}$. At $70^{\circ}$ and $80^{\circ}$ the light intensity becomes stronger and the maximum tends toward the center of the cylinder. It appears that the fuel comes from the cylinder wall at the top. A possible explanation is that unburned fuel desorbs from crevices as the piston travels downward. At $130^{\circ}, 140^{\circ}$, and $280^{\circ}$ the exhaust valve is open and the combustion products leave. The darkening in these pictures indicates the reduction of $n_{\text {iso-C } 8 \mathrm{H} 18}(x, y)$. It also verifies that the fluorescence originates inside the cylinder and is not some unknown background or scattered light.

Figures 8 and 9 show that some dynamics of the processes are quite different from cycle to cycle. Figure 8 shows a series of pictures, all at $330^{\circ}$, in which fuel is starting to come in. The differences among the pictures shows clearly the fluctuations in the fuel density that are caused by the turbulent inlet process. Figure 9 is a similar series at $26^{\circ}$ after the ignition (CA

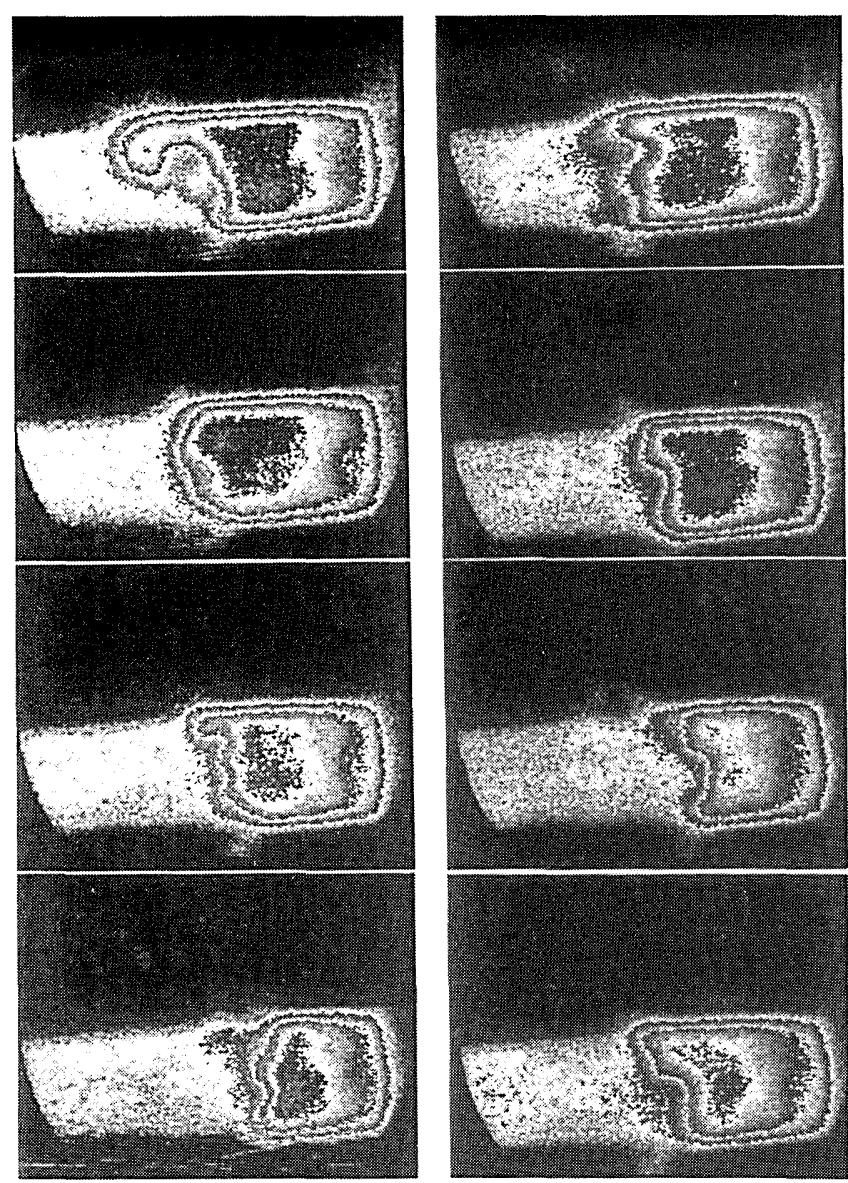

Fig. 9. Density distributions of isooctane in succeeding cycles at $\mathrm{CA}=20^{\circ}$ (i.e., during combustion). The intensity scale is the same as in Fig. 7.

$=20^{\circ}$ ). Analogous fluctuations in the combustion are seen from the variations of the flame front positions.

\section{B. OH Fluorescence Without a Laser via Normal Fluorescence}

The flame front is made visible by a simpler technique (snapshot without a flashbulb) than that used above. The laser is not used and pictures are taken of the normal $\mathrm{OH}$ fluorescence (NF) caused by the combustion itself. The camera's gate was lengthened to 1 $\mu \mathrm{s}$.

The sequence in Fig. 10 is similar to that in Fig. 7 . The pictures start $\approx 4^{\circ}$ after the plug fires. Most pictures are at $2^{\circ}$ intervals, which at $1000 \mathrm{rpm}$ corresponds to $\approx 2 \times 10^{4} \mu \mathrm{s}$. This means that the $1-\mu \mathrm{s}$ time resolution is still excellent. The flame front spreads from the spark plug through the cylinder. Initially the brightest light is around the plug and then migrates to the right.

This migration is caused by the projection of the spherical flame front. Initially the front expands toward the piston, and the cylinder's head and its left wall. When it reaches these, further movement occurs to the right. 

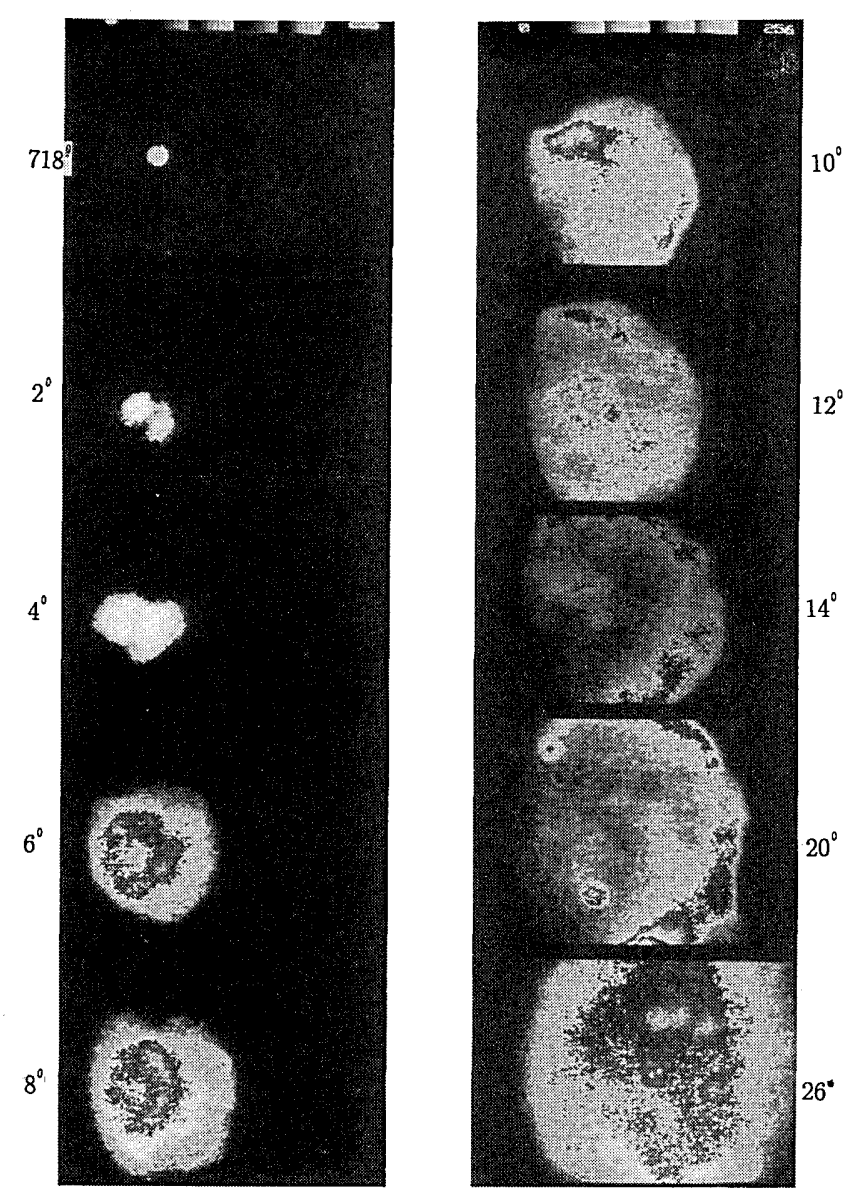

Fig. 10. Natural fluorescence of $\mathrm{OH}$ as a function of CA. The flame front spreads out from the spark plug.

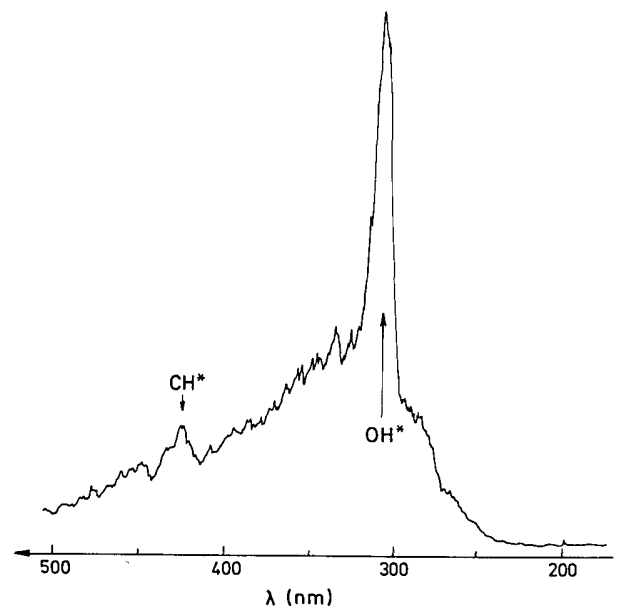

Fig. 11. Dispersion spectrum of the natural $\mathrm{OH}$ fluorescence at $\mathrm{CA}$ $=20^{\circ}$.

A dispersion spectrum of the NF is shown in Fig. 11. Its most prominent feature is the $(A \rightarrow X), 0-0$ band, $\mathrm{OH}$ emission around $310 \mathrm{~nm}$ that occurs exclusively in the flame front ${ }^{14}$ and so provides another handle to locate the front. We remove the other spectral feature near $430 \mathrm{~nm}$, that probably originates from $\mathrm{CH}$, with

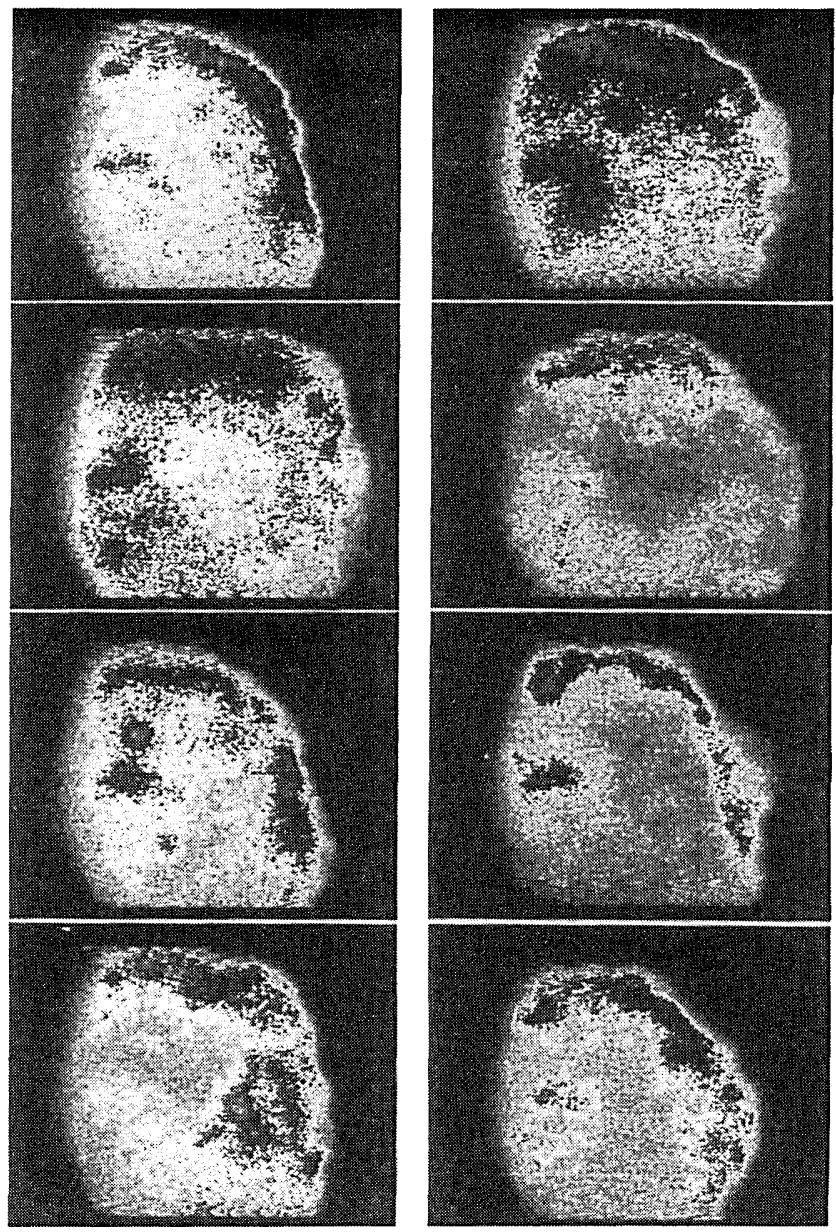

Fig. 12. Natural fluorescence of $\mathrm{OH}$ from different cycles at $\mathrm{CA}=$ $20^{\circ}$. Note the fluctuation in the flame front locations.

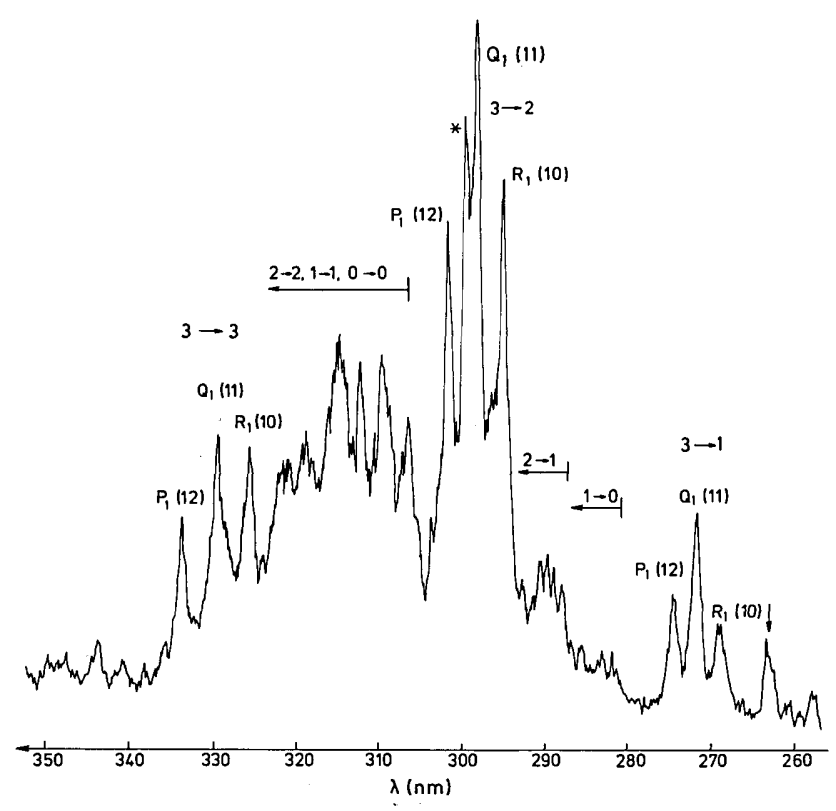

Fig. 13. Dispersion spectrum of $\mathrm{OH}$ produced by $\mathrm{KrF}$ excitation via the $Q_{1}(11)$ transition of the $0-3$ band. The * is the unknown resonance and the vertical arrow is a Raman line. 
the Schott UG11 camera filter that transmits around $340 \mathrm{~nm}$.

Figure 12 shows a series of pictures of the NF, all at $26^{\circ}$ after ignition. This again shows the variation in the flame front's location among succeeding cycles.

\section{State-Selective Detection of OH via LIPF}

The LIPF approach to the state-selective detection of $\mathrm{OH}$ is that shown on the right-hand side of Fig. 1 and was discussed earlier, i.e., 248-nm excitation in the 0-3 band of the $X \rightarrow A$ electronic transition.

Figure 13 shows a dispersion spectrum with the laser tuned to the $Q_{1}(11)$ line. The groups of lines around 270,295 , and $330 \mathrm{~nm}$ correspond to $X \leftarrow A$ emission from $v^{\prime}=3$ to $v^{\prime \prime}=1,2$, and 3 , respectively. There are other, less structured bands originating from $v^{\prime} \leq 2$ and these involve quenching to other vibrational and rotational states. This spectrum is similar to that from an open butane flame and was discussed ${ }^{2}$ for that case. A point made in Ref. 2 is that the integrated intensities for those quenched molecules greatly overstate their importance because (a) they have larger Franck-Condon factors and (b) the much shorter lifetime of $v^{\prime}=3$ reduces its fluorescence. Surprisingly the ratio of fluorescence from $v^{\prime}=3$ to that from $v^{\prime} \leq 2$ is quite similar in the $\approx 25$-bar engine and in the 1-bar open flame.

In addition to the known $\mathrm{OH}$ spectrum, Fig. 13 also has a peak at $297 \mathrm{~nm}$ that is discussed in Appendix A as the unknown resonance.

Figure 14 is an $\mathrm{OH}$ excitation spectrum. It is similar to that from an open $\mathrm{H}_{2}-\mathrm{O}_{2}$ flame. ${ }^{2}$ However the ratios of rotational lines originating from $N^{\prime \prime}=10$ and 11 to those from $N^{\prime \prime}=8$ are larger in the engine, probably because of its higher temperature.

The signal with the laser beam off should be compared with the valleys between the peaks. This indicates that the $\mathrm{OH}$ measurements at $25 \mathrm{bar}$ in an engine can be made with a background of $<5 \%$ and without disturbance from other constituents. The spectrum's SNR is very good, in spite of the turbulent combustion.

The group of six pictures at the left of Fig. 15 shows $n_{\mathrm{OH}}(x, y)$ that are selective for $v^{\prime \prime}=0, N^{\prime \prime}=8$ at two crank angles. The pictures are with the laser tuned (left) to the $P_{2}(8)$ transition and with the laser detuned (right), i.e., in a valley of Fig. 14. The difference represents $n_{\mathrm{OH}}(x, y)$ in the selected state. The middle, at $20^{\circ}$, and the top, at $80^{\circ}$, are during and after the passage of the flame front, respectively.

There should not be any $\mathrm{OH}$ ahead of the flame front. In the upper pictures the flame front has already passed through the cylinder and so $\mathrm{OH}$ might be anywhere. The middle pictures show $n_{\mathrm{OH}}(x, y)$ when the flame front is still in the picture. At the right, the cometlike trails are $\mathrm{NF}$, which was previously measured with a 1- $\mu$ s camera gate. Here, to minimize the contribution of NF, we reduce the gate to $200 \mathrm{~ns}$.

There is still considerable light at the middle right, where no $\mathrm{OH}$ is expected. This is broadband fluorescence from unburned fuel, as described above. A com-

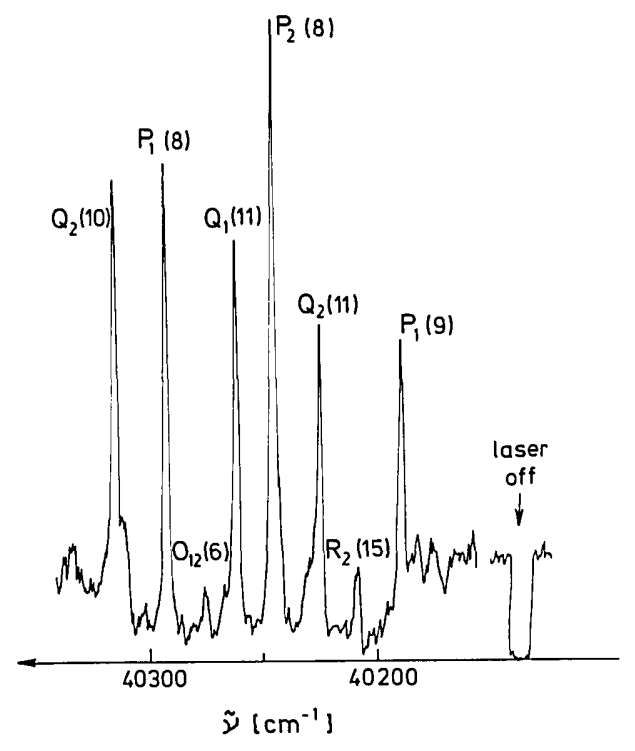

Fig. 14. $\mathrm{OH}$ excitation spectrum at $\mathrm{CA}=30^{\circ}$ with the monochromator at $329.5 \pm 0.5 \mathrm{~nm}$ (comparatively narrow transmission).

parison of the left and right pictures in Fig. 15 shows that $\mathrm{OH}$ is, as expected, behind the flame front.

An appropriate filter might separate the $\mathrm{OH}$ light from most of the fuel light. We tried a filter that transmitted around $300 \mathrm{~nm}$. Although this is not optimal for OH, a glance at the spectra in Figs. 6 and 13 shows that it does discriminate against the fuel's LIF. The bottom of Fig. 15 has the same condition as its middle but with the filter. It clearly helps. There was not time to optimize the filtering. However the excitation spectrum in Fig. 14 shows that, with the use of the narrow bandwidth of the monochromator, the background intensities are $<5 \%$ of those at a resonance. This means it should be possible to suppress most emission from all other species.

For these reasons, $\mathrm{OH}$ is particularly well suited for temperature-field measurements in the engine. These were attempted with the arrangement in Fig. 4, and some preliminary data were obtained, but the time limitation prevented completion of the measurement.

Felton et al. ${ }^{7}$ and Schipperijn et al. ${ }^{8}$ used LIF for $\mathrm{OH}$. They excited via the 1-0 band and measured the 1-1 band fluorescence so that their laser and detection wavelengths were well separated. Our LIPF measurements also have a large wavelength separation. Felton et al. worked at 13.8 bar and found that the $0-0$ emission was comparable with that from 1-1, which indicates that extensive vibrational energy transfer occurs. Suntz et $a l .{ }^{15}$ reported LIF in $\mathrm{OH}$ in an enginelike environment with excitation and fluorescence via the $0-0$ band. That approach was limited to $<7.5$ bar because the Rayleigh scattering became much more intense than the fluorescence. Our data are up to 30 bar.

A comparison of five different LIF (not LIPF) methods for analysis of $\mathrm{OH}$ at $\approx 0.1$ bar has been recently presented by Laurendeau and Goldsmith. ${ }^{16}$ 


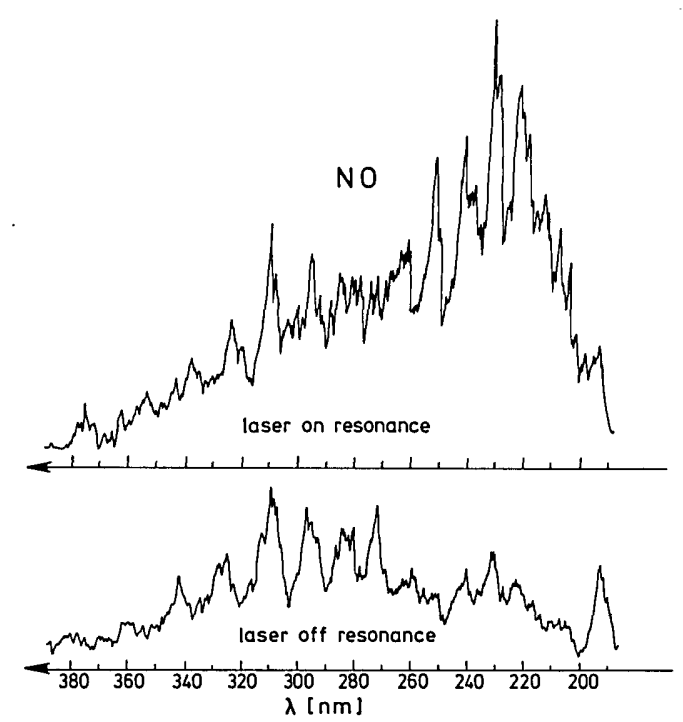

Fig. 16. Top, dispersion spectrum of NO produced by ArF excitation from $1 \rightarrow 0, X \rightarrow D$ via the $R_{1}(26)$ and $Q_{1}(32)$ overlapping transitions. Bottom, the laser is tuned off that transition.

\section{Detection of NO via LIF}

An important result of this section is that NO could be detected with high sensitivity in the cylinder through $\approx 75 \%$ of the engine cycle. The importance of the minimization of $\mathrm{NO}_{x}$ formation in combustion is well known.

We previously described ${ }^{13}$ NO detection by $193 \mathrm{~nm}$. An obvious requirement of the engine analysis is that most of the laser light go through the cylinder. We monitored the transmitted light. The cylinder was transparent (even in the high pressure region) until ignition occurs at $714^{\circ}$, but from there to $\approx 150^{\circ}$ the light was completely absorbed. Apparently some combustion intermediates absorb strongly and light transmission is not restored until their density is sufficiently reduced. The detection of NO was particularly good for CA $>180^{\circ}$.

The detection of NO in the engine works as well as in a flame. We first tune the laser to a peak that excites from $v^{\prime \prime}=1$ in the $X$-state to $v^{\prime}=0$ in the $D$-state. That peak includes contributions mainly from $R_{1}(26)$ and $Q_{1}(32)$, and a small amount from $P_{1}(39)$. We then record a dispersion spectrum. Figure 16 has this spectrum, as well as the same one when the laser is off this resonance. The difference is caused by the vibrationally selected NO.

Figure 17 has an excitation spectrum of NO. The assignments ${ }^{13}$ shown there indicate that the spectrum is completely due to the 1-0 band of NO. This means that vibrationally selected NO can be analyzed in an automobile cylinder.

Figure 15 and 18 show pictures of $n_{N O}(x, y)$. Figure 18 compares the fluorescence when the laser is on- or off-resonance. The difference between the two pictures is due to the state-selected NO. Obviously the influence of other constituents is negligible.

The right-hand side of Fig. 15 has three pictures of

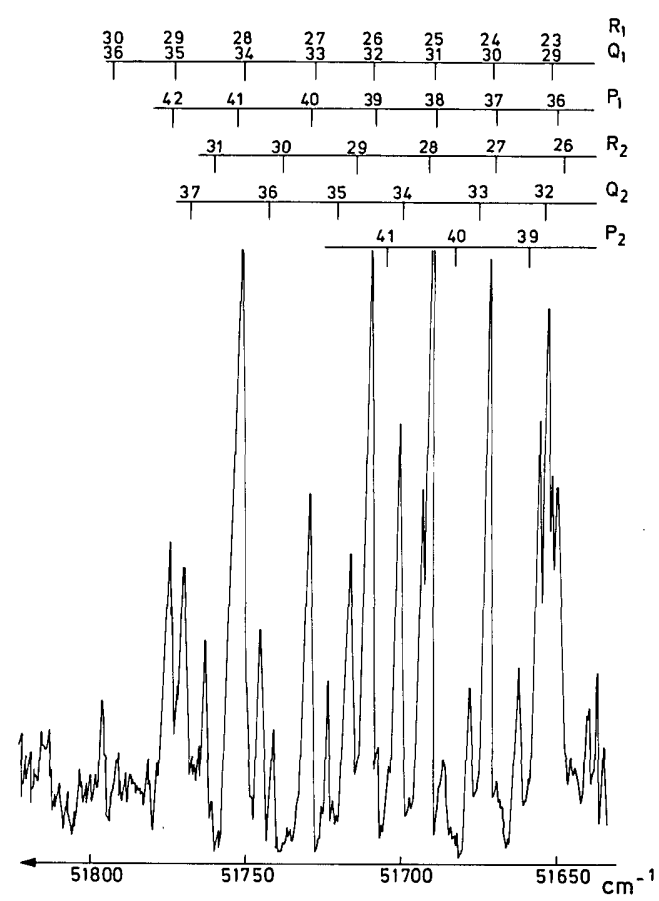

Fig. 17. NO excitation spectrum at $\mathrm{CA}=170^{\circ}$ with the monochromator at $208 \pm 1.5 \mathrm{~nm}$. This spectrum is from the $1-0, X-D$ band. See also Ref. 13.
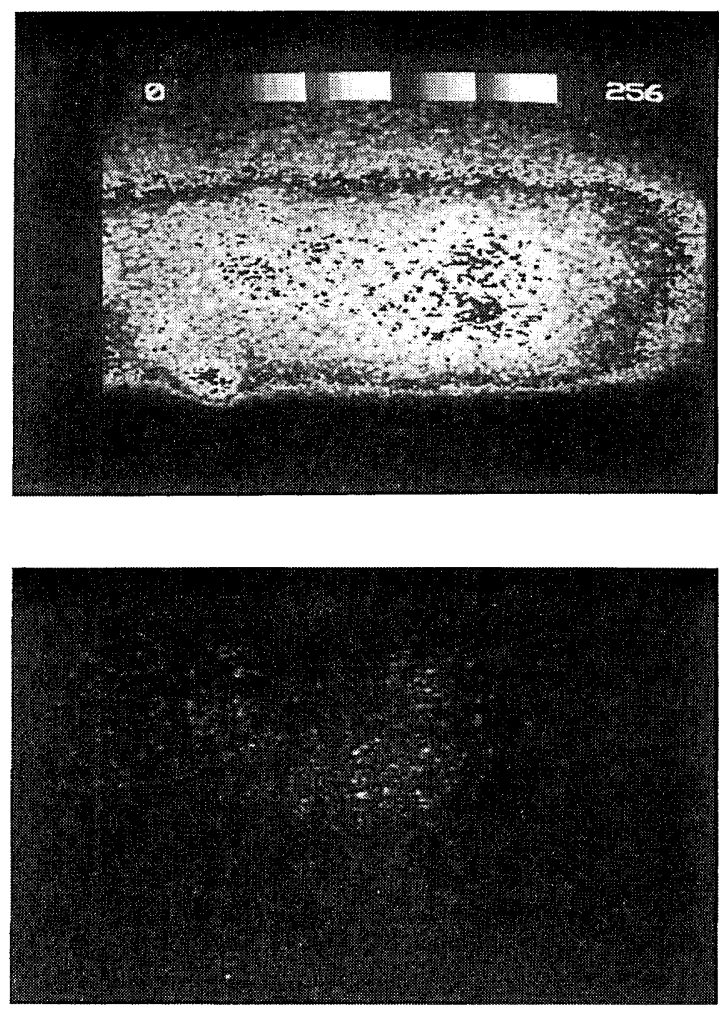

Fig. 18. Density distributions for state-selected NO $\left(v^{\prime \prime}=1\right)$ at CA $=170^{\circ}$. The top and bottom pictures are for the laser tuned on or off the transition, respectively. 


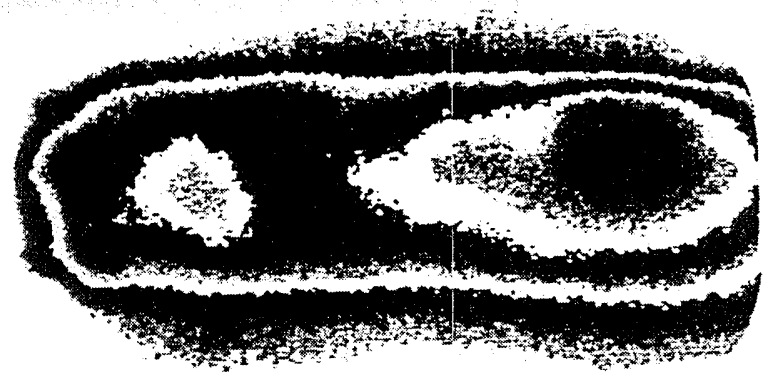

Fig. 19. Average of ten density distributions for state-selected NO $\left(v^{\prime \prime}=1\right)$ at $\mathrm{CA}=200^{\circ}$ from different cycles.

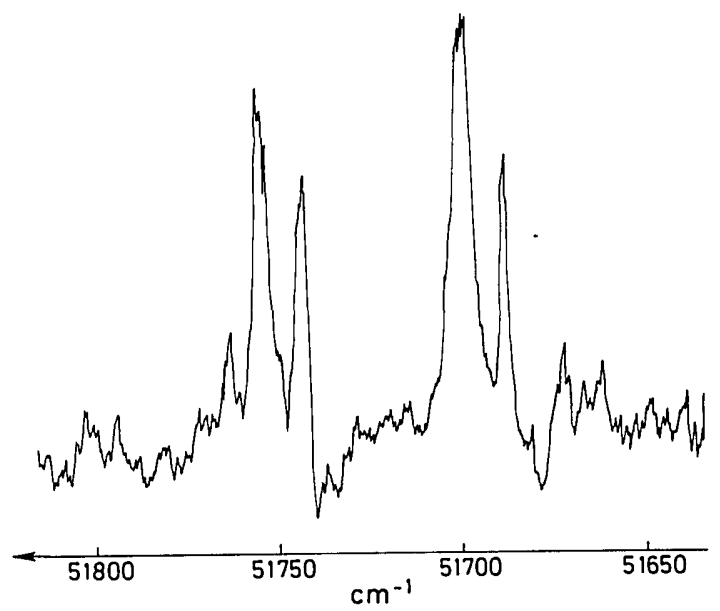

Fig. 20. $\mathrm{O}_{2}$ excitation spectrum at $\mathrm{CA}=200^{\circ}$ with the monochromator at $201 \pm 0.5 \mathrm{~nm}$.

NO with identical conditions, at $200^{\circ}$, from successive cycles. It is immediately obvious that there are large differences. Some cycles produce much more NO than others. Further, the NO densities are greater at the spark plug (left), in the middle, and at the right cylinder edge. Figure 19 has an average of ten pictures like those in Fig. 15 and that also shows the regions of large NO densities.

\section{E. State-Selective Detection of $\mathrm{O}_{2}$ via LIPF}

There are $\mathrm{O}_{2}$ transitions within the 193-nm tuning range that allow analysis for many rotational states within the $v^{\prime \prime}=0-3$ vibrational states. Figure 20 displays an excitation spectrum that is about the same as that from an open flame ${ }^{12}$ and so is not discussed further here.

Figure 21 has pictures for $\mathrm{O}_{2}$, in $v^{\prime \prime}=2, J^{\prime \prime}=19$, with the laser on- and off-resonance. The difference is the state-selective distribution $n_{\mathrm{O}_{2}}(x, y)$ and is determined at $20 \mathrm{bar}$. This distribution is similar to that of the fuel shortly before ignition. The diminishing intensity toward the right is caused by Beer's law absorption of the laser beam.

The spectroscopy and $n_{\mathrm{O}_{2}}(x, y)$ at different CA will not be discussed further here. However it should be
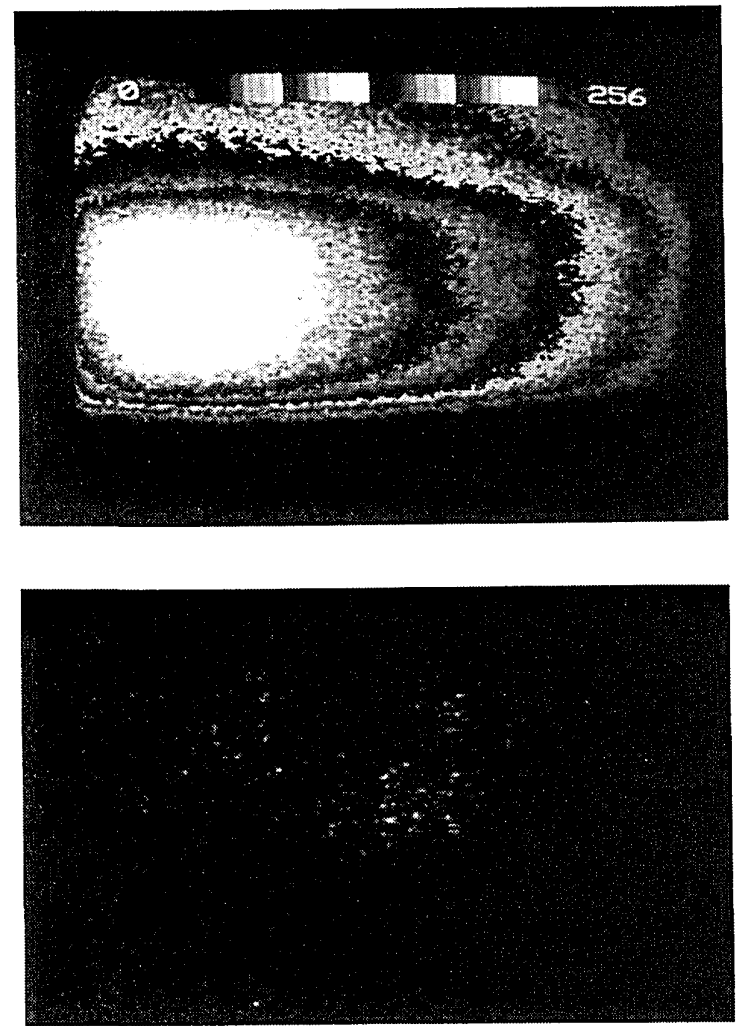

Fig. 21. Density distributions for state-selected $\mathrm{O}_{2}\left(v^{\prime \prime}=2, J^{\prime \prime}=19\right)$ at $\mathrm{CA}=700^{\circ}$. The top and bottom pictures are on and off the resonance, respectively. The pressure is $\approx 20 \mathrm{bar}$.

noted that such state-selective detection for oxygen can be used to obtain temperature fields. In addition, one could get information about the combustion through simultaneous fuel- $\mathrm{O}_{2}$ measurements.

\section{Concluding Remarks}

These results are from three weeks of measurements in a cylinder of an engine that is similar to those in mass-produced cars. This is the first application of these methods to such an engine. The results include 2 -D pictures that make many gasdynamic and chemical processes visible. They show time-dependent variations of constituent densities as well as their cyclic fluctuations. We discussed some basic spectroscopy that should be useful for future combustion work of this type. Many potential worries about the method were either groundless or could be avoided.

The high spectral brightness of the excimer lasers and their tunability provide adequate and selective illumination, respectively. The correct filters then serve to avoid the problems with Rayleigh scattering and with emission from other states or species. Thus the cameras see very selective pictures that are obtained on an essentially instantaneous time scale.

The main results of the investigation are summarized here. (1) With the use of LIF, the fuel, isooctane, is observed in the intake of the fuel-air mixture, in the ignition, in the movement of the flame front, and in the decrease of its density in the exhaust 
phase. (2) The natural fluorescence from $\mathrm{OH}$ very clearly defines the flame front. (3) The LIPF allows $\mathrm{OH}$ to be measured, state selectively, in extreme engine combustion conditions and in the presence of high pressures. (4) An analogous LIPF state-selective determination of oxygen was also successful at $20 \mathrm{bar}$. (5) LIF also makes the distribution of NO visible in the cylinder, although not during the combustion phase.

Spectroscopic results also help to improve the system. They are needed to find the best laser tuning frequencies and optimal filter specifications so that selected molecules or specific states can be measured without interference.

This work shows that LIPF 2-D analyses, although they are still in the developmental stage, provide a superb state and species selectivity. They should become quantitative after suitable calibration.

Quenching will badly confuse normal LIF and natural fluorescence analyses if the aim is to have quantitative results. However qualitative pictures may be completely acceptable for many applications.

An important aspect for practical engine development is that the density distributions of particular species can be seen directly on a TV monitor. That means that the results of changing engine or fuel parameters can be seen in real time. The turbulent nature of the processes in the cylinder are important and they can be very nicely visualized with this procedure. Whether quantitative measurements via LIPF are needed or whether qualitative pictures via LIF will suffice depends on the particular needs of the user.

Erhard Rothe thanks H. Pauly, Director at the MPI, for the opportunity to work at his institute and the NSF for partial support.

\section{Appendix A: Unknown Resonance}

The $\mathrm{OH}$ dispersion spectrum in Fig. 13 shows a peak, that is marked with * and is called the unknown resonance, between the three $\mathrm{OH}$ emission lines in the 2-3 band. Figure 22 shows an excitation spectrum with the monochromator set (and narrowed to $\approx 1 \mathrm{~nm}$ ) to allow a maximum selection of this transition. It has a distinct structure, but it also has some $\mathrm{OH}$ lines that are transmitted by the monochromator. One of its peaks is marked with the *. Figure 23 shows another dispersion spectrum with the laser set to the * resonance. It has two strong peaks at 275 and $300 \mathrm{~nm}$ and some nearby subsidiary structure. Although this resonance is not understood, it (a) shows that new spectroscopic features can be studied in an automobile cylinder and (b) that one must be careful to exclude such phenomena when identifying specific states.

\section{Appendix B: Brief Comparison of CARS, LIF, and LIPF}

The CARS method can also be used to measure $n_{i}$ and $T$. However it can only find these at specific locations. In particular, in turbulent systems, spatial distributions are of greater interest. Figure 9 nicely illustrates this point. A preselected point might lie ahead of the flame front in one cycle, and behind in

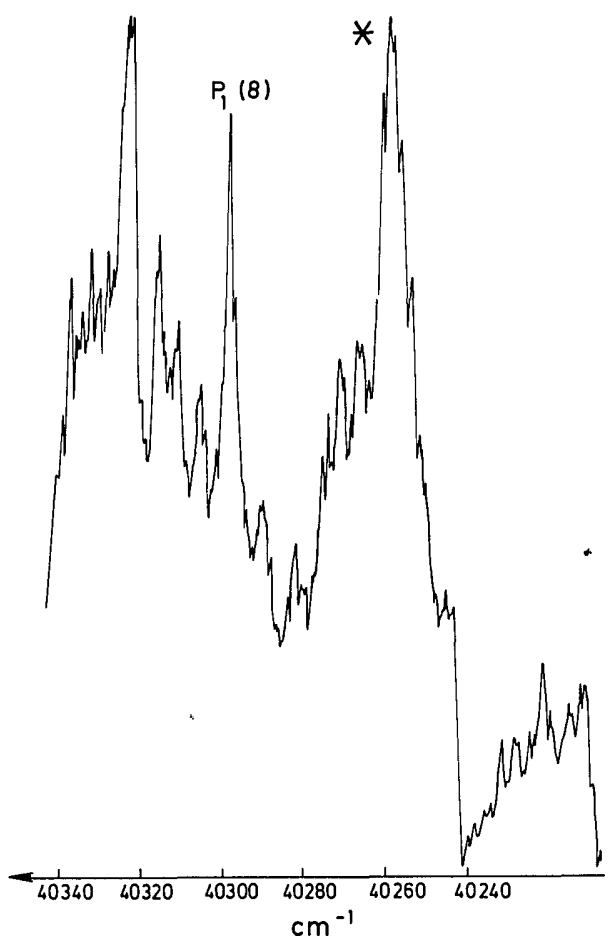

Fig. 22. Excitation spectrum for the unknown resonance at $\mathrm{CA}=$ $80^{\circ}$ with the monochromator at $300 \pm 0.5 \mathrm{~nm}$. The * indicates the laser setting for Fig. 23.

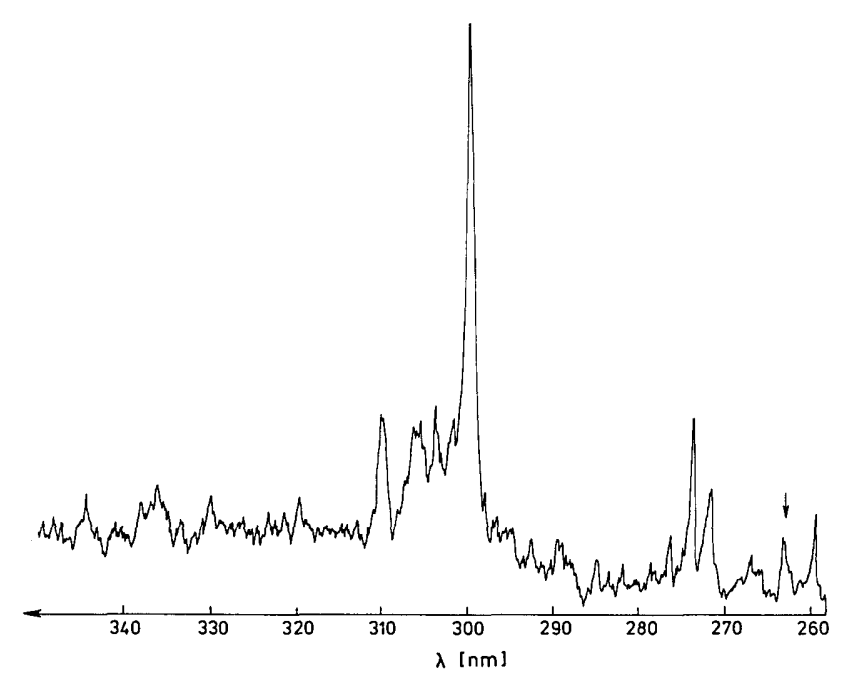

Fig. 23. Dispersion spectrum from the unknown resonance. The laser frequency is given in the caption of Fig. 22. The arrow is a Raman line.

another. That means that there will be large fluctuations in the measurement. Even for an average over many shots, the mean that is obtained is difficult to interpret. A big advantage of LIF is that 2-D images can be obtained from a single shot. Averaging over many shots can badly blur details in turbulent systems that are inherently nonreproducible. And with the addition of LIPF, quantitative, calibrateable measure- 
ment systems should be developed in a straightforward way.

A brief summary of the main points relevant to combustion analyses with these methods follows.

(1) CARS has an extensive literature. ${ }^{1}$ The experimental setup is reasonably complex, is difficult to analyze, and it has modest sensitivity. As discussed above, it is limited to point measurements and thus cannot obtain the single shot distributions that are essential for analyses of turbulent systems.

(2) Normal LIF also has an extensive literature, a simple experimental arrangement, and easy data analysis (at low pressures). It has much better sensitivity than CARS. Instantaneous density distributions can be found at low pressures. However because of quenching, neither temperature nor absolute density can be measured at high pressures $(>\approx 0.01$ bar $)$, nor can their distributions, because $Q$ varies with location. However it will yield qualitative pictures that may be useful for many purposes, including on-line visualization.

(3) LIPF has about the same experimental ease and sensitivity as normal LIF but can be applied at high pressures. As shown here, instantaneous (time scale $\approx 20 \mathrm{~ns}$ ) density distributions can be measured at high pressures. Temperature fields are also measurable. Further, absolute temperature and density calibrations are possible.

Clearly for quantitative measurement of turbulent high pressure phenomena, LIPF is the method of choice if suitable spectroscopy is available for the desired molecule.

\section{References}

1. See, e.g., A. C. Eckbreth, Laser Diagnostics for Combustion Temperature and Species (Abacus Press, Cambridge, MA, 1988).

2. P. Andresen, A. Bath, W. Gröger, H. W. Lülf, G. Meijer, and J. J. ter Meulen, "Laser-Induced Fluorescence with Tunable Excimer Lasers as a Possible Method for Instantaneous Temperature Field Measurements at High Pressures: Checks with an Atmospheric Flame," Appl. Opt. 27, 365-378 (1988).

3. G. A. Massey and C. J. Lemon, "Feasibility of Measuring Temperature and Density Fluctuations in Air Using Laser-Induced $\mathrm{O}_{2}$ Fluorescence," IEEE J. Quantum Electron. QE-20, 454-457 (1984).

4. M. P. Lee, P. H. Paul, and R. K. Hanson, "Laser-Fluorescence Imaging of $\mathrm{O}_{2}$ in Combustion Flows Using an ArF Laser," Opt. Lett. 11, 7 (1986); "Quantitative Imaging of Temperature Fields in Air Using Planar Laser-Induced Fluorescence of $\mathrm{O}_{2}$," Opt. Lett. 12, 75-77 (1987); L. M. Cohen, M. P. Lee, P. H. Paul, and R. K. Hanson, "Two-Dimensional Imaging Measurements in Supersonic Flows Using Laser-Induced Fluorescence of Oxygen," AIAA-87-1527, AIAA Twenty-Second Thermophysics Conference, Honolulu (8-10 June 1987).

5. G. Laufer, R. L. McKenzie, and W. M. Huo, "Radiative Processes in Air Excited by an ArF Laser," Opt. Lett. 13, 99-101 (1988).

6. G. Kim, L. M. Hitchcock, G. P. Reck, and E. W. Rothe, "LIF from Predissociating States Using Tunable ArF Excimer Light," Bull. Am. Phys. Soc. 34, 1382-1382 (1989).

7. P. G. Felton, J. Mantzaras, D. S. Bomse, and R. L. Woodin, "Initial Two-Dimensional Laser Induced Fluorescence Measurements of OH Radicals in an Internal Combustion Engine," in Proceedings, International Fuels and Lubricants Meeting, Portland OR, 10-13 Oct. 1988 (Society of Automotive Engineers, Warrendale, PA 15096), paper 881633.

8. F. W. Schipperijn, R. Nagasaka, R. F. Sawyer, and R. M. Green, "Imaging of Engine Flow and Combustion Processes," in Proceedings, International Fuels and Lubricants Meeting, Portland OR, 10-13 Oct. 1988 (Society of Automotive Engineers, Warrendale, PA 15096), paper 881631.

9. R. P. Lucht, "Optical Engineering," in Laser Spectroscopy and Its Applications, L. J. Radziemski, R. W. Solarz, and J. A. Paisner, Eds. (Marcel Dekker, New York, 1987), Chap. 9.

10. A. M. Wodtke, L. Hüwel, H. Schlüter, H. Voges, G. Meijer, and P. Andresen, "Predissociation of $\mathrm{O}_{2}$ in the $B$ State," J. Chem. Phys. 89, 1929-1935 (1988).

11. G. Meijer, J. J. ter Meulen, P. Andresen, and A. Bath, "Sensitive Quantum State Selective Detection of $\mathrm{H}_{2} \mathrm{O}$ and $\mathrm{D}_{2} \mathrm{O}$ by $(2+1)$ Resonance Enhanced Multiphoton Ionization," J. Chem. Phys. 85, 6914-6922 (1986); V. Engel, G. Meijer, A. Bath, P. Andresen, and R. Schinke, "The C-A Emission in Water: Theory and Experiment," J. Chem. Phys. 87, 4310-4314 (1987).

12. G. Meijer, A. M. Wodtke, H. Voges, H. Schlüter, and P. Andresen, "State Selective Detection of CO Using a Tunable ArF Excimer Laser," J. Chem. Phys. 89, 2588-2589 (1988).

13. A. M. Wodtke, L. Hüwel, H. Schlüter, G. Meijer, P. Andresen, and H. Voges, "High-Sensitivity Detection of NO in a Flame Using a Tunable ArF Laser," Opt. Lett. 13, 910-912 (1988). Figure 2 of this paper has some misassigned and missing lines. We thank Michel Versluis, at the University of Nijmegen, for finding this error and Thomas Seelemann, at MPI fur Strömungsforschung, for identifying the lines in our Fig. 17.

14. A. G. Gaydon, The Spectroscopy of Flames (Chapman \& Hall, London, 1957).

15. R. Suntz, H. Becker, P. Monkhouse, and J. Wolfrum, "Two Dimensional Visualization of the Flame Front in an Internal Combustion Engine by Laser Induced Fluorescence of OH Radicals," Appl. Phys. B 47, 287-293 (1988).

16. N. M. Laurendeau and J. E. M. Goldsmith, "Comparison of Hydroxyl Concentration Profiles Using Five Laser-Induced Fluorescence Methods in a Lean Subatmospheric-Pressure $\mathrm{H}_{2}$ / $\mathrm{O}_{2}$ /Ar Flame," Combust. Sci. Technol. 63, 139-152 (1989). 